\title{
Better Late Than Never - An Analysis of Last-Minute Travelers Attending a Specialist Travel Medicine Clinic in Ireland
}

\author{
Gerard Thomas Flaherty ${ }^{1,2^{*}}$, Muhammad Haziq Hasnol', Lokman Hakim Sulaiman ${ }^{3,4}$ \\ ${ }^{1}$ School of Medicine, National University of Ireland Galway, Galway, Ireland \\ ${ }^{2}$ School of Postgraduate Studies, International Medical University, Kuala Lumpur, Malaysia \\ ${ }^{3}$ Institute for Research, Development and Innovation, International Medical University, Kuala Lumpur, Malaysia \\ ${ }^{4}$ Department of Community Medicine, School of Medicine, International Medical University, Kuala Lumpur, Malaysia
}

Corresponding Author: Gerard Thomas Flaherty, MD, Professor, School of Medicine, National University of Ireland Galway, Galway, Ireland. Tel: +353-91495469, Email: gerard.flaherty@nuigalway.ie

Received August 3, 2019; Accepted November 3, 2019; Online Published December 15, 2019

\begin{abstract}
Introduction: Last-minute travelers (LMTs) are a vulnerable group, because it may not be possible to adequately vaccinate them against exposure to infectious diseases. The purpose of this retrospective cross-sectional study was to describe the characteristics of LMTs attending a travel health clinic.

Methods: The following data was extracted from records of travelers attending the Tropical Medical Bureau (Galway, Ireland) over a 6-year period with less than 2 weeks remaining before their departure: gender, age, occupation, destination(s), purpose of travel, departure date, travel duration, travel group size, accommodation, past medical history, medications, and vaccination history.

Results: Of 7555 traveler records, $1296(17.2 \%)$ were of LMTs, of whom 45 (3.5\%) were recurrent LMTs. LMTs were equally likely to be male or female. The mean age of this cohort was 32.2 years. The most common travel destination was Asia, and holiday was the most frequent purpose of travel. The mean interval before departure was $7.54 \pm 3.65$ days, and the mean travel duration was $7.36 \pm 2.3$ weeks. The majority $(n=454,35.1 \%)$ of LMTs traveled in pairs. Approximately 2 in $5(n=497,38.4 \%)$ travelers reported a past medical history; over half ( $n=674,52.0 \%$ ) had previously received travel vaccinations. The majority $(n=1202,92.8 \%)$ of LMTs were unable to complete a scheduled course of pre-travel vaccines.

Conclusion: This study provides insight into the characteristics and travel patterns of LMTs. A large proportion of LMTs have pre-existing medical conditions. Further research should focus on the travel health risk-taking behavior of these individuals.

Keywords: Travel, Travel Medicine, Vaccination, Chronic Illness
\end{abstract}

Citation: Flaherty GT, Hasnol MH, Sulaiman LH. Better late than never - An analysis of last-minute travelers attending a specialist travel medicine clinic in Ireland. Int J Travel Med Glob Health. 2019;7(4):123-128. doi:10.15171/ijtmgh.2019.26.

\section{Introduction}

Travelers who enquire about the optimal time to attend a pre-travel health consultation are typically advised to access pre-travel services at least one month before their intended departure date to enable courses of travel vaccines to be completed and to ensure that they have protective immunity by the time they reach their destination. Previous generations of travelers who had no access to modern technological advantages may have invested considerably more time in their travel itinerary preparations than current travelers do. Indeed, very high levels of travel itinerary uncertainty exist, and this can have a potentially negative impact on the effectiveness of the travel medical consultation. ${ }^{1}$
According to the generally accepted but arbitrary definition, the last-minute traveler (LMT) attends a consultation with fewer than two weeks remaining before departure, ${ }^{2}$ although a recently published study of LMTs in the United States employed a definition of seven days or fewer. ${ }^{3}$ Some LMTs, such as humanitarian aid workers, may have to leave at even shorter notice, even within days or hours. ${ }^{4}$ There is typically only enough time for a single clinical encounter before they travel overseas.

LMTs presenting for a pre-travel consultation are a vulnerable group, because it may not be possible to adequately protect them against exposure to infectious disease owing to a lack of time. It may also be challenging for practitioners to

Copyright $\odot 2019$ The Author(s). This is an open-access article distributed under the terms of the Creative Commons Attribution License (http:// creativecommons.org/licenses/by/4.0), which permits unrestricted use, distribution, and reproduction in any medium, provided the original work is properly cited. 
offer other elements of pre-travel health counseling which form part of a normal personalized pre-travel risk assessment ${ }^{5}$ as a result of traveler uncertainty about the itinerary. Limited knowledge exists about their demographic characteristics and the features of their planned trips. LMTs comprised $16 \%$ of all travelers presenting to a network of US clinical practices over a 6-year period. ${ }^{3}$ Knowledge of these factors may enable travel medicine practitioners to design strategies to promote earlier pre-travel care and to better anticipate the needs of LMTs attending their clinics. This is among the first studies to analyze LMT patterns in clinical practice and the first to be conducted outside the United States.

\section{Methods}

A retrospective cross-sectional study was performed. Paper records of all travelers attending the Tropical Medical Bureau in Galway, Ireland (2013-2018) with less than 2 weeks remaining before departure were retrieved and interrogated. This clinic specializes in travel medicine with a focus on the pre-travel health preparation of travelers of all ages, many with complex itineraries and medical histories. While the majority of individuals self-refer to the clinic, they may also be referred by their general practitioner. Each individual traveler is allotted 20 minutes for a consultation. The time is increased to 30 minutes and above for a pair or group of travelers, depending on the complexity of their planned trip.

The clinic is staffed by multiple physicians with training in travel medicine. Vaccines are administered by specialist travel health nurses, and pre-travel advice is typically reinforced by the nursing staff on subsequent clinic visits when the traveler returns for booster vaccine doses. Approximately 1200 travelers attend the clinic each year. They complete a traveler registration card prior to their medical consultation, and data from that card is entered by the physician into an electronic health record. The travelers' most recent clinic visit is linked to all of his previous consultations at this clinic. A printout of the electronic health record based on the consultation also accompanies each traveler's record card and provides information on which vaccines, if any, were administered to the LMT.

The following anonymized data was extracted and entered into a database created in Microsoft Excel 2013: gender, age, occupation, destination(s), purpose of travel, departure date, travel duration, travel group size, accommodation, past medical history, medications, and travel vaccination history. Data was summarized as percentages and means with standard deviations for the purposes of descriptive analysis. This study satisfied the requirements of the local clinical research ethics committee.

\section{Results}

Of 7555 traveler records examined, 1296 (17.2\%) were LMTs, of whom $45(3.5 \%)$ were recurrent LMTs. The characteristics of the recurrent LMTs are summarized in Table 1. Of all the LMTs, 614 (8.1\% of total number of travelers) were traveling within 7 days or less. LMTs were equally likely to be male $(\mathrm{n}=655)$ or female $(\mathrm{n}=641)$. The mean age of LMTs was 32.2 $(+/-13.8)$ years. Students $(n=261,20.1 \%)$ and professionals
Table 1. Characteristics of Recurrent Last-Minute Travelers

\begin{tabular}{lc}
\hline Characteristic & \\
\hline Gender, No. (\%) & $27(60)$ \\
Male & $18(40)$ \\
Female & $43.5(+/-13.4)$ \\
Mean age (y, +/- SD) & \\
Travel destination, No. (\%) & $15(33)$ \\
Asia & $18(40)$ \\
Africa & $1(2)$ \\
Europe & $2(4)$ \\
North America & $12(27)$ \\
Latin America & 0 \\
Oceania & \\
Purpose of trip, No. (\%) & $30(67)$ \\
Holiday & $9(20)$ \\
Business & $6(13)$ \\
Other & \\
Past medical history, No. (\%) & $19(42)$ \\
Yes & $26(58)$ \\
No & $2.36(+/-0.61)$ \\
\hline Mean number of trips as LMT (+/- SD) &
\end{tabular}

LMT: last minute traveler; SD: standard deviation.

$(n=269,20.8 \%)$ comprised similar proportions of the overall group of LMTs. The remaining travelers belonged to other occupational groups, were unemployed or retired, but this data was not specifically captured in this study.

The most common travel destination was Asia (Figure 1). Holiday ( $n=991,76.5 \%$ ) was the most frequent purpose of travel, but $15.8 \%(n=205)$ of LMTs were engaged in business travel. The majority of LMTs planned to stay in hotel accommodations $(n=975,75.2 \%)$, with $19.8 \%$ of LMTs $(\mathrm{n}=256)$ staying in hostels and $1.5 \%(\mathrm{n}=19)$ exclusively camping on their upcoming trip. The mean interval before departure was $7.54(+/-3.65)$ days, and the mean travel duration was $7.36(+/-2.3)$ weeks. The majority $(n=454$, $35.1 \%$ ) traveled in pairs. The total annual number of LMTs peaked in 2016 with 308 travelers (Figure 2). The months with the highest incidence of LMTs (Figure 3$)$ were June $(n=171)$, July $(n=153)$, and August $(n=116)$. A total of $497(38.4 \%)$

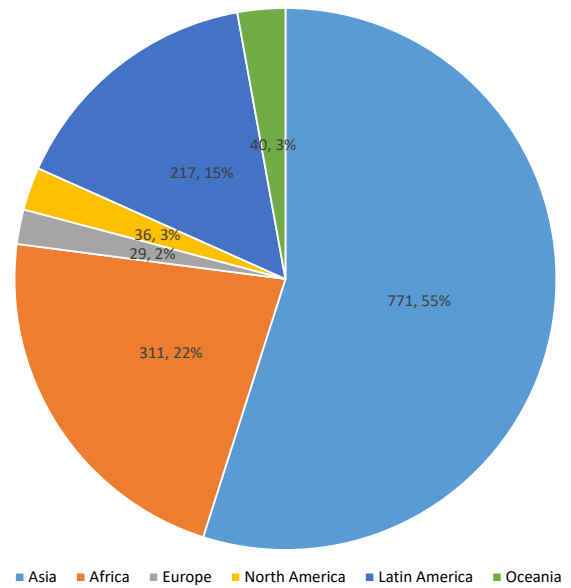

Figure 1. Last-Minute Regional Traveler Destinations. 


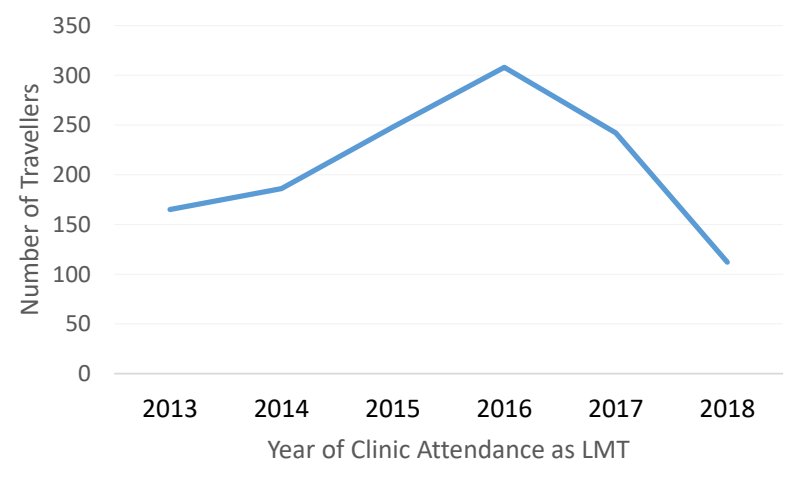

Figure 2. Temporal Trends in Last-Minute Traveler Clinic Attendances.

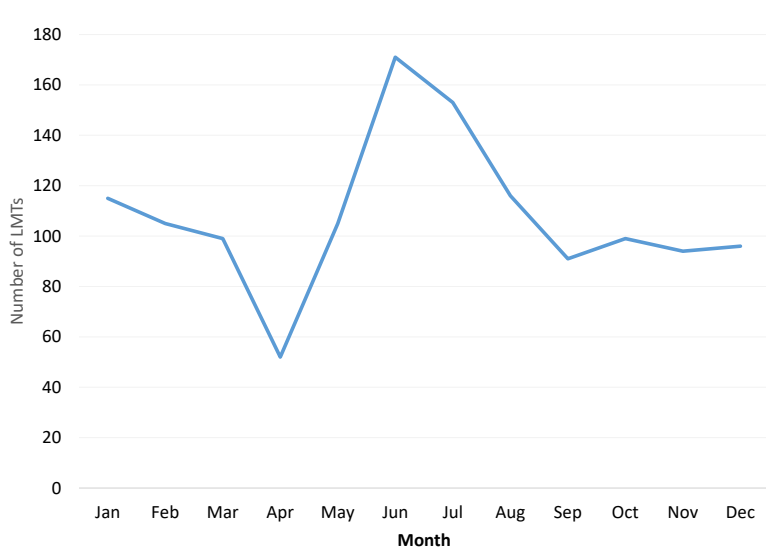

Figure 3. Seasonality of Last-Minute Traveler Clinic Attendance.

travelers reported a past medical history (Table 2). The most commonly documented medical conditions were hay fever $(\mathrm{n}=170)$, insect bite sensitivity $(\mathrm{n}=104)$, cardiovascular disease $(n=95)$, respiratory disease $(n=91)$, and allergies $(n=70)$.

A total of 674 (52.0\%) travelers had received previous travel vaccinations. Of the 1258 LMTS who were prescribed at least one travel vaccine at their most recent consultation, the vast majority $(n=1165,92.6 \%)$ received single dose vaccines. Of the 528 LMTs planning to visit yellow fever-endemic countries, $231(43.8 \%)$ were administered a yellow fever vaccine at the clinic. No data was recorded in relation to the requirement by International Health Regulations for yellow fever vaccine for the LMTs' current trip. The vast majority $(n=1202,92.8 \%)$ of LMTs were unable to receive a course of travel vaccines at their index consultation, because the time remaining before their planned departure date was insufficient.

\section{Discussion}

The current study focused on the characteristics of LMTs who referred to the clinic for a pre-travel health consultation with less than 2 weeks remaining before their departure date. Over 1 in 6 travelers at the clinic satisfied the criteria for last-minute travel, with just under 1 in 25 of these travelers having attended the clinic previously as an LMT. The Global TravEpiNet Consortium studied this phenomenon across
Table 2. Past Medical History of Last-Minute Travelers

\begin{tabular}{lc}
\hline Medical Condition & No. $(\%)$ \\
\hline Hay fever & $170(13.1)$ \\
\hline Insect bite sensitivity & $104(8.0)$ \\
\hline Cardiovascular disease & $95(7.3)$ \\
Respiratory disease & $91(7.0)$ \\
\hline Allergies & $70(5.4)$ \\
\hline Photosensitivity & $38(2.9)$ \\
\hline Psychiatric disorder & $18(1.4)$ \\
\hline Infectious jaundice & $16(1.2)$ \\
\hline Previous surgery & $10(0.8)$ \\
\hline Diabetes mellitus & $10(0.8)$ \\
\hline Thyroid disease & $9(0.7)$ \\
\hline Neurological disorder & $9(0.7)$ \\
\hline Skin condition & $8(0.6)$ \\
\hline Gastrointestinal disease & $7(0.5)$ \\
\hline Reproductive problems & $5(0.4)$ \\
\hline Musculoskeletal condition & $4(0.3)$ \\
\hline Ear, nose and throat disease & $2(0.2)$ \\
\hline
\end{tabular}

their diverse network of clinics in the USA and reported a prevalence of last-minute travel of $16 \%$ using the definition of 7 days or fewer. ${ }^{3}$ Applying this definition to the sample in the current study, a lower prevalence of $8.1 \%$ was recorded. It would be of interest to investigate the occurrence of lastminute travel among general practices in Ireland, as this may differ from that observed in the specialist travel health clinic setting.

The motivation LMTs is likely to vary across different clinical practices. Clinics who advise a higher proportion of humanitarian aid workers or business travelers, for example, may expect to encounter more LMTs, as these individuals are often deployed to their destinations on shorter notice. The Global TravEpiNet Consortium showed a higher prevalence of last-minute travel among business and VFR travelers, ${ }^{3}$ while 3 in 4 of the LMTs in the current study were traveling for leisure purposes. Some recreational travelers may book holidays at short notice to avail themselves of last-minute commercial deals, and employees may not be able to confirm their annual leave with sufficient advance notice to enable earlier travel planning. With the greater use of self-booked travel using commercial websites rather than travel agents, the modern traveler may not benefit from the prompts which travel agents are more likely to provide in relation to pre-travel health care. ${ }^{6}$ Student travelers were well represented in the current sample. They may be subject to restrictions on clinic attendance due to learning placements and examination schedules, making last-minute travel more likely. Students are a vulnerable group of often inexperienced travelers who may be less risk averse and more adventurous ${ }^{7,8}$ and whose study abroad activities may expose them to significant infectious risks. ${ }^{9}$

Despite a mean interval before departure of just over a week in the present study, the LMTs were planning to stay for an average of nearly two months at their destination. This is in accordance with the findings of the American study which found that LMTs were more likely than non-LMTs to 
spend at least one month overseas. ${ }^{3}$ This extended exposure to destination travel health risks is of concern, given that LMTs may not be able to benefit from full protection against vaccine-preventable tropical infectious diseases. Most of the LMTs in the present study did not have a recurrent pattern in the studied clinic of last-minute travel, but analysis of this subgroup provided interesting observations. Recurrent LMTs in this study were more likely to be middle-aged males traveling to Africa for a holiday. Given the burden of health risks associated with travel to sub-Saharan Africa and the requirement in some African countries for evidence of Yellow Fever vaccine having been administered at least 10 days prior to arrival, even if traveling from non-endemic countries, this finding was unexpected. One approach to recurrent lastminute travel is to offer pre-emptive travel vaccinations to protect travelers against anticipated exposures during future trips. ${ }^{4}$ Some travelers may present as LMTs because they have previously received travel vaccines. In the current study, just over half of the LMTs had evidence of previous travel vaccines. Although it was not possible to record this data, it could be that these previous vaccines no longer provided cover if booster doses had not been received. In some cases, the traveler may have been under the false impression that having already received single dose vaccines appropriately for a previous trip, courses of vaccines against hepatitis $B$, rabies, and Japanese encephalitis were not indicated for the current trip.

One of the authors (GTF) routinely counsels travelers to attend future pre-travel consultations with at least 4-6 weeks remaining before departure in order to allow enough time for protective immunity to develop from the primary administration of single dose vaccines and from a series of vaccines. This study was not designed to capture the individualized decision-making that applied in each consultation. It is conceivable that some LMTs appropriately attended with as little as a week or 2 remaining before departure, having been informed that only a booster dose of the hepatitis A or typhoid vaccine, for example, was recommended for their index trip. Travel medicine research has already embraced an intriguing era of vaccinomics where, based on an individual traveler's specific genetic susceptibility to infectious diseases, some vaccines may be withheld if the risk of disease does not merit their administration. ${ }^{10}$ With the advent of approved alternative vaccine regimens with shorter intervals between doses for protection against rabies ${ }^{11,12}$ or Japanese encephalitis, ${ }^{13}$ some LMTs may at least be able to receive their final booster dose prior to an upcoming trip.

It is accepted travel medicine practice to recommend that a LMT who cannot complete a vaccine course before departing seek out a reliable source of the same or an equivalent vaccine locally. ${ }^{4}$ Whether this will be possible will depend to some extent on the quality and ease of access to medical care at the travel destination and the capacity of the individual traveler to prioritize this activity having just arrived at their destination. Providing incomplete protection against diseases requiring a series of vaccines may be problematic when the traveler misinterprets the clinician's advice and exposes himself unnecessarily to infectious risks at the destination. In some cases where the index trip is short, where the risk assessment predicts a low level of likely exposure, and where the traveler has imminent plans for future travel, it may be sensible to commence a travel vaccine series prior to last-minute travel schedule follow-up clinic visits for booster doses upon one's return. This must be accompanied by unambiguous advice about the lack of protection afforded during the index trip and the need to follow specific travel health advice.

While no data on the subject is available herein, it is important when dealing with LMTs to observe strictly the International Health Regulations in relation to completion of the International Certificate of Vaccination or Prophylaxis. Medico-legally, it would be indefensible to provide false reassurance to an LMT about the likelihood of gaining entry into a yellow fever-endemic country where there is a requirement to present evidence of vaccination at the border, but fewer than 10 days have elapsed since administration of the vaccine. ${ }^{14}$ The same applies to the use of the meningococcal vaccine in Hajj pilgrims to Mecca. In the case of a last-minute, extended-stay traveler who plans to remain at the destination for longer than a year, the travel medicine clinician should strongly recommend a consultation in the host country. ${ }^{4}$ If vaccine courses cannot be started or completed prior to the trip, they can be given by a local practitioner upon arrival. With the ease of use of atovaquone-proguanil or doxycycline, LMTs should not be disadvantaged in relation to malaria chemoprophylaxis unless a lack of time prevents them from collecting their prescription before they travel. If this is a potential scenario, the traveler should be strongly discouraged from purchasing over-the-counter antimalarial drugs overseas, given the high level of counterfeit drugs in circulation in many countries. ${ }^{15}$

In the current study, an equal likelihood of being a LMT was observed in both males and females, while the study by Yates et al demonstrated a male preponderance. ${ }^{3}$ The mean age of travelers in the current study (32 years) was comparable to the median age of travelers in the US study ( 34 years). That the older traveler is less likely to engage in last-minute travel is suggested by both the current study and that of Yates et $\mathrm{al},{ }^{3}$ but this question deserves further prospective study. This would be a worthy research topic for the newly formed Older Traveler Interest Group of the International Society of Travel Medicine (ISTM). ${ }^{16}$ The most common last-minute travel destination in the current study was Asia; a previous study also showed that this was the most frequently visited destination by travelers living in Ireland. ${ }^{17}$ Whether the behavior of LMTs is influenced by their perceived risk in relation to their destination and whether they have traveled there on a previous occasion is worthy of further research, but it was beyond the scope of the current study.

The LMTs in the current study were more likely to be accompanied by a traveling companion. The presence of an organized, well-prepared travel companion may lull some travelers into a false sense of security if they assume a more passive role in the travel preparations, but this hypothesis cannot be confirmed from the results of the current study. Apart from vaccination pressures, LMTs with chronic medical illnesses such as obesity ${ }^{18}$ or HIV $^{19}$ face the additional 
challenge of leaving enough time to allow adjustments to their management to take effect and for identifying reliable sources of competent medical care before they travel abroad. LMTs with chronic conditions, those who are taking regular medications, and those who have complex itineraries involving exposure to high altitudes may not have adequate time to take a test dose of acetazolamide for prophylaxis of high-altitude illness, for example. ${ }^{20}$

In the clinic in this study, booster doses of vaccines are administered by a travel health nurse who reinforces the travel health advice given at the medical consultation. The LMT cannot benefit from this opportunity. A previous study of rabies prevention advice highlighted the rapid decay of information received at the initial consultation. ${ }^{21}$ This has implications for medication compliance and travel risk behaviors in LMTs.

\section{Limitations of Study}

This retrospective study is limited by its design. A future prospective study would be desirable. The researchers were constrained by the data included on the travel vaccination records, and the reasoning behind individual vaccine choices could not be captured. In the absence of reliable annual clinic attendance data, the researchers were unable to interpret the longitudinal trends in LMTs attending the studied clinic. Moreover, there was no intention to compare the non-LMTs with the LMTs in this study. A comparison may have yielded valuable additional information. Last-minute travel has been a neglected topic in the travel medicine literature despite being included in the ISTM Body of Knowledge. ${ }^{22}$ Future research should investigate the rationale and motivation of last-minute travel in an attempt to better understand the phenomenon and to design strategies which would discourage last-minute travel in a targeted campaign. Web-based travel companies should routinely remind clients at the time of booking to arrange a pre-travel health consultation and leave adequate time to complete all recommended and required vaccines.

\section{Conclusion}

In conclusion, LMTs are a vulnerable group who are at increased risk of vaccine-preventable diseases if they have insufficient time to develop protective immunity and, in particular, if they are unable to complete a vaccine series prior to travel. The current findings suggest that LMTs often have chronic medical conditions and embark on holidays of moderate duration to high-risk destinations, despite presenting late for pre-travel consultations. This study provides an insight into the characteristics and travel patterns of LMTs. The findings may help efforts to access travelers who might otherwise be unable to complete courses of travel vaccinations for diseases including hepatitis B, rabies, and Japanese encephalitis.

\section{Authors' Contributions}

GTF conceived the study in collaboration with LHS. GTF was responsible for the study design. MHH and GTF completed the data collection. Data interpretation and analysis was conducted by GTF and MHH with input from LHS. GTF prepared the first draft of the manuscript which was reviewed

\section{Research Highlights}

\section{What Is Already Known?}

Last-minute travelers (LMTs) are a vulnerable group because it may not be possible to adequately vaccinate them against exposure to infectious diseases.

\section{What This Study Adds?}

This study provides an insight into the characteristics and travel patterns of LMTs, who are typically in their early 1930s and traveling to Asia in groups for leisure. A large proportion of LMTs have pre-existing medical conditions. The current findings will aid efforts to access travelers who might otherwise be unable to complete courses of travel vaccinations for diseases including hepatitis $\mathrm{B}$, rabies, and Japanese encephalitis. Further research should focus on the travel health risk-taking behavior of these individuals.

for significant intellectual content by MHH and LHS. All authors read and approved the final version of the manuscript.

\section{Conflict of Interest Disclosures}

The authors declare no conflicts of interest.

\section{Ethical Approval}

The protocol for this study was approved by the Research Ethics Joint Committee of the International Medical University, Kuala Lumpur, Malaysia.

\section{Funding/Support}

$\mathrm{MHH}$ received an unrestricted educational bursary from the Travel Medicine Society of Ireland to support his involvement in this study.

\section{References}

1. Flaherty G, Md Nor MN. Travel itinerary uncertainty and the pre-travel consultation--a pilot study. J Travel Med. 2016;23(1). doi:10.1093/jtm/tav010.

2. Zuckerman JN, Van Damme P, Van Herck K, Löscher T. Vaccination options for last-minute travellers in need of travel-related prophylaxis against hepatitis A and B and typhoid fever: a practical guide. Travel Med Infect Dis. 2003;1(4):219-226. doi:10.1016/j. tmaid.2003.10.001

3. Yates JA, Rao SR, Walker AT, et al. Characteristics and preparation of the last-minute traveler: analysis of vaccine usage in the Global TravEpiNet Consortium. J Travel Med. 2019;26(6). doi:10.1093/ jtm/taz031.

4. Rosselot GA. Last-minute travelers. In: Brunette GW, Nemhauser JB, eds. CDC Yellow Book 2020: Health Information for International Travel. New York: Oxford University Press; 2019:19-21.

5. Flaherty GT. One size does not fit all: towards personalized risk assessment in travel medicine. J Travel Med. 2018;25(1). doi:10.1093/jtm/tay076.

6. Flaherty G, Lim D, Fry G. Travel agency awareness of the health risks of international travel; a pilot study. Int J Travel Med Glob Health. 2015;3(2):45-48. doi:10.20286/ijtmgh-030268.

7. Hartjes LB, Baumann LC, Henriques JB. Travel health risk perceptions and prevention behaviors of US study abroad students. J Travel Med. 2009;16(5):338-343. doi:10.1111/j.17088305.2009.00322.x.

8. Heywood AE, Zhang M, Maclntyre CR, Seale H. Travel risk 
behaviours and uptake of pre-travel health preventions by university students in Australia. BMC Infect Dis. 2012;12:43. doi:10.1186/1471-2334-12-43.

9. Johnston N, Sandys N, Geoghegan R, O'Donovan D, Flaherty G. Protecting the health of medical students on international electives in low-resource settings. J Travel Med. 2018;25(1). doi:10.1093/ $\mathrm{jtm} / \mathrm{tax} 092$.

10. Torresi J, Steffen R. Redefining priorities towards graded travelrelated infectious disease research. J Travel Med. 2017;24(6). doi:10.1093/jtm/tax064.

11. Soentjens P, Andries P, Aerssens A, et al. Preexposure intradermal rabies vaccination: a noninferiority trial in healthy adults on shortening the vaccination schedule from 28 to 7 days. Clin Infect Dis. 2019;68(4):607-614. doi:10.1093/cid/ciy513.

12. World Health Organization (WHO). WHO expert consultation on rabies: third report. WHO Technical Report Series, No. 1012. Geneva: WHO; 2018.

13. U.S. Food and Drug Administration. Package Insert and Patient Information—Ixiaro. https://www.fda.gov/media/75777/download. Accessed 23 November 2019.

14. Kennedy KM, Flaherty GT. Medico-legal risk, clinical negligence and the practice of travel medicine. J Travel Med. 2016;23(5). doi:10.1093/jtm/taw048.

15. Brower V. Counterfeit and substandard malaria drugs in Africa.
Lancet Infect Dis. 2017;17(10):1026-1027. doi:10.1016/s14733099(17)30528-5.

16. Flaherty GT, Rossanese A, Steffen R, Torresi J. A golden age of travel: advancing the interests of older travellers. J Travel Med. 2018;25(1). doi:10.1093/jtm/tay088.

17. Han CT, Flaherty G. Profile of travelers with preexisting medical conditions attending a specialist travel medicine clinic in Ireland. J Travel Med. 2015;22(5):312-317. doi:10.1111/jtm.12221.

18. Flaherty GT, Geoghegan R, Brown IG, Finucane FM. Severe obesity as a barrier to international travel: a qualitative analysis. J Travel Med. 2019;26(3). doi:10.1093/jtm/taz018.

19. Chang L, Lim BCW, Flaherty GT, Torresi J. Travel vaccination recommendations and infection risk in HIV-positive travellers. J Travel Med. 2019;26(6). doi:10.1093/jtm/taz034.

20. Flaherty GT, Kennedy KM. Preparing patients for travel to high altitude: advice on travel health and chemoprophylaxis. $\mathrm{Br} J \mathrm{Gen}$ Pract. 2016;66(642):e62-64. doi:10.3399/bjgp16X683377.

21. Flaherty G, Gouda J, Fry G. Awareness of rabies risk in a sample of travelers attending an Irish travel medicine clinic. Int J Travel Med Glob Health. 2015;3(2):53-57. doi:10.20286/ijtmgh-030262.

22. Flaherty GT, Lim Yap K. Bibliometric analysis and curriculum mapping of travel medicine research. J Travel Med. 2017;24(5). doi:10.1093/jtm/tax024. 